\title{
Antifungal susceptibility pattern and identification of Candida species in women of child-bearing age suffering from malaria, typhoid and diabetes in Nsukka, Nigeria
}

\author{
Emmanuela I. Nwoko ${ }^{1}$, Anthony C. Mgbeahuruike ${ }^{2,3^{*}}$, Ejike E. Ugwuijem ${ }^{3}$ and \\ Onwumere S. Idolor ${ }^{4}$
}

${ }^{1}$ Department of Human Kinetics and Physical Education, Public Health Unit, Faculty of Education, University of Nigeria, Nsukka, Nigeria.

${ }^{2}$ Department of Veterinary Microbiology and Pathology, Faculty of Veterinary Medicine, University of Nigeria, Nsukka, Nigeria.

${ }^{3}$ Department of Microbiology, Faculty of Biological Sciences, University of Nigeria, Nsukka, Nigeria.

${ }^{4}$ Department of Agricultural Technology, School of Agriculture, Delta State Polytechnic, Ozoro, Delta State, Nigeria.

Received 24 November, 2018; Accepted 11 April 2019

\begin{abstract}
Candidiasis in women of child-bearing age is a major problem in most hospitals in Nigeria. High vaginal swabs were analyzed for Candida presence in women of child bearing age using Chromogenic agar. Isolated Candida spp. were analyzed phylogenetically to trace their evolutionary relatedness. Growth rate assay was done using Sabouruand and Dextrose medium supplemented with $\mathrm{NaCl}$ and glucose. The resistance pattern of the Candida spp. was tested using fluconazole and extracts (methanol and ethyl) from Byrsocarpus coccineus. Out of the 79 fungal samples analyzed, $21(26.6 \%)$ were $C$. tropicalis, $25(31.6 \%)$ were C. krusei, $10(12.7 \%)$ were C. parapsilosis and $8(10.1 \%)$ were C. albicans. About $15(19.0 \%)$ had mixed morphology on CHROM agar Candida and could not be identified. The healthy women presented very low percentage (8.9\%) of Candida species. The sequenced Candida spp. had $100 \%$ sequence identity to $C$. tropicalis. High concentration of glucose $(1 \mathrm{~g} / \mathrm{ml})$ and $\mathrm{NaCl}(0.5 \mathrm{M})$ decreased the growth of the fungus. In conclusion, $C$. tropicalis and $C$. krusei were more prevalent in the malaria patients. $C$. albicans were found mostly in the typhoid patients. The plant extracts showed lower antifungal activity in comparison with fluconazole but they showed a huge antifungal potential.
\end{abstract}

Key words: Byrsocarpus coccineus, Candida, diabetes, fluconazole, malaria, phylogeny, typhoid.

\section{INTRODUCTION}

Vaginal candidiasis is a very common health problem in both immunocompetent and immunocompromised individuals, while oral candidiasis is much more common in immunocompromised individuals (Denning et al., 2018; Muzny and Schwebke, 2015; Fidel, 2002). Candida colonization of the vaginal and oral mucosa occurs in

${ }^{*}$ Corresponding author. E-mail: anthony.mgbeahuruike@unn.edu.ng.

Author(s) agree that this article remain permanently open access under the terms of the Creative Commons Attribution License 4.0 International License 
healthy women of all ages, over $75 \%$ of women will experience at least one vaginal infection during their lifetime (Vermitsky et al., 2008). Prevalence studies indicate that 10 to $55 \%$ of healthy and asymptomatic women have vaginal cultures positive for C. albicans (Muzny and Schwebke, 2015). C. albicans is the most frequently isolated Candida spp. in human candidiasis (more than 50\%) and it has been reported to be the most pathogenic species of the genus Candida (Ben-Ami, 2018; Deorukhkar and Saini, 2015). However, other Candida spp., like C. glabrata (10 to $30 \%)$, C. tropicalis (10 to $20 \%$ ), C. parapsilosis (10 to $20 \%$ ) have been reported. Vaginal colonization with Candida in women has been reported to be due to among other factors, regular use of antibiotics (Canela et al., 2018). The use of antibiotics has been reported to cause destruction of bacterial flora which creates a more favorable environment for Candida overgrowth (Canela et al., 2018). It is therefore apparent that Candida infections mainly occur as opportunistic infections due to altered conditions of the host. Human infections by Candida spp. are usually endogenous because Candida is frequently part of the normal flora of the mouth, throat, large intestine, vagina and the skin of humans (Shao et al., 2007). However, infections are more common in patients whose immune systems are compromised (Kim and Sudbery, 2011). Sexual transmission from patients with vaginitis to their sexual partners has been reported (Murray et al., 2002). Most Candida spp. have inherent resistance to available antifungal azoles. In C. albicans, development of secondary resistance to fluconazole treatment is commonly encountered in HIV-infected patients (Pereira et al., 2010). Among the non-albicans species, C. tropicalis and C. parapsilosis appear to be susceptible to azoles but $C$. tropicalis is less susceptible to fluconazole than C. albicans. However, C. glabrata is intrinsically more resistant to most antifungal agents, particularly to fluconazole. C. krusei is also resistant to fluconazole. C. Iusitaniae which accounts for 1 to $2 \%$ of all candidemia is susceptible to azoles, but has a higher intrinsic resistance to amphotericin B (Cruciani and Serpelloni, 2008). Studies have reported the emergence of antifungal-resistant $C$. albicans fungemia in bone marrow transplant recipients being administered longterm fluconazole prophylaxis (Cornely et al., 2012; Alexander et al., 2013). However, the number of cases of fluconazole-resistant candidemia caused by $C$. albicans in patients with cancer who are receiving azole prophylaxis still remains small (Cornely et al., 2012; Alexander et al., 2013). C. krusei is innately resistant to fluconazole, in addition to intrinsic resistance, nearly about $20 \%$ strains of C. glabrata can acquire resistance during the course of therapy (Deorukhkar et al., 2014). C. tropicalis is generally considered as a fluconazolesusceptible species. However, recent studies have documented emergence of fluconazole resistance (Deorukhkar et al., 2014; Silva et al., 2012). C. parapsilosis is reported to have high minimum inhibitory concentration to echinocandins (Deorukhkar and Sani, 2016). For treatments in both human and animals, therapeutic options become limited when there is cross resistance to all antifungal azoles.

Therefore, medicinal plants may provide an alternative to antifungal drugs due to the increasing resistance towards the available azoles. Byrsocarpus coccineus is an indigenous tropical plant found in West Africa (Kossivi et al., 2014). Previous studies have reported considerable antimicrobial activities of the extracts of $B$. coccineus on C. albicans, Escherichia coli, Salmonella typhi and Staphylococcus aureus (Ahmadu et al., 2006). The antimicrobial property of $B$. coccineus has been reported to be due to a coumaryl derivative and flavonoid glycoside isolated from its leaves (Akindele and Adeyemi, 2006). Other phytochemical constituents like tannins, sugar and saponins have been reported to contribute to its antimicrobial activity (Ahmadu et al., 2006). B. coccineus has also been used in the treatment of diarrhea (Akindele and Adeyemi, 2006). Although B. coccineus has been shown to be a promising candidate for antifungal therapy, no study has compared its efficacy with the commercially available antifungal azoles.

In the present study, a comparative analysis of Candida spp from high vaginal swab (HVS) of healthy women (20 to 30 years) with normal clinical conditions was made with the Candida spp. isolated from HVS of women within the same age group with clinical conditions such as malaria, typhoid and diabetes. Also, the resistance pattern of the Candida spp was investigated using the common antifungal agent fluconazole and extracts from the medicinal plant, $B$. coccineus. The result from this work will give an insight into the relationship between Candida infections in women and some common clinical conditions in the tropics as well as give insight on the potentials of using this plant as a replacement for common azoles that are no more efficacious in treatment due to resistance.

\section{MATERIALS AND METHODS}

\section{Sample collection}

The study was conducted at the Daughters' of Devine Love (DDL) Hospital, Ehalumona, Nsukka. Female outpatients 20 to 30 years of age with asymptomatic candidiasis without any history of antifungal, antibiotic and antimalarial therapy for the past one month were enrolled in the study. Patients were diagnosed with diabetes on clinical presentation by a certified endocrinologist and clinically confirmed by a satisfied physician to be suffering from either malaria or typhoid. Potential participants were approached in the clinic waiting room and consent granted. Clinical samples of high vaginal swabs (HVS) were self-collected by the female patients. Swab sticks of about $3 \mathrm{~cm}$ in length were self-inserted into the upper part of the vaginal lumen and gently rotated. The swab stick was then withdrawn carefully to avoid contamination with the external genitalia and immediately inserted into its plastic pack, labeled with the patient's identification tag, age and medical history 
and was quickly transported to the laboratory for further processing. In parallel, samples of HVS of healthy females (females without any history of illness or any history of medication of any kind) within the same age bracket were collected from female students living in Awolowo Hall of the University of Nigeria Nsukka as control. The study protocol was approved at each of the study sites by the local institutional review board. There were 100 female participants in this trial.

\section{Fungal isolation and morphological characterization on CHROM agar Candida}

Each of the samples was inoculated into petri plates of Sabouraud dextrose agar (SDA) (Oxoid, Basingstoke, United Kingdom) containing $5 \mathrm{mg} / \mathrm{ml}$ of chloramphenicol and incubated at $28^{\circ} \mathrm{C}$ under aerobic condition for $48 \mathrm{~h}$. Single colonies of the obtained cultures were transferred into freshly prepared SDA, incubated for another $48 \mathrm{~h}$ to produce pure cultures. Individual colonies from the pure cultures were inoculated into plates of CHROM agar Candida/differential media (CM10002 BRILLIANCE ${ }^{\text {TM }}$ CANDIDA AGAR; Oxoid UK) reconstituted with $70 \%$ ethanol. The fungi were grouped together based on their colours on the CHROM agar Candida and were later classified to different Candida species based on colour following already existing information in the literature (Daef et al., 2014).

\section{Sequencing the fungal internal transcribed spacer region}

To confirm the result from the screening on CHROM agar Candida, molecular identification was carried out on one isolate from the $C$. tropicalis group. From a 3 day old SDA (Oxoid, Cambridge, UK) culture grown at $25^{\circ} \mathrm{C}$, genomic DNA was extracted using hexadecyltrimethylammonium bromide (CTAB)-based method (Nygren et al., 2008). The concentration of the extracted DNA was determined spectrophotometrically using NanoDrop (Thermo Scientific, Wilmington, DE). PCR amplification of the highly conserved fungal internal transcribed spacer (ITS) region was done using primer pairs ITS1F and ITS4 (Gardes and Bruns, 1993; White et al., 1990) as previously described (Kårén et al., 1997). Amplified PCR products were purified using ethanol-sodium acetate precipitation protocol and sequencing was performed by Macrogen Europe (Amsterdam, The Netherland).

\section{Phylogenetic analysis}

The generated sequence was submitted to GenBank at the National Center for Biotechnology Information (NCBI) with the accession number MK101318, and used for BLASTN analysis at NCBI. Additional ITS sequences from Candida spp. were retrieved from GenBank at NCBI. Sequence alignment was performed using MUSCLE algorithm (Edgar et al., 2004). Phylogenetic analysis was performed using neighbour-joining method implemented in MEGA ver. 5.0 (Tamura et al., 2011). The analysis was run using maximum composite likelihood substitution model with uniform rates among sites and homogeneous patterns among lineages. Gaps were deleted in pairwise comparisons and statistical support for the phylogenetic grouping was assessed by bootstrap analysis using 1000 replication.

\section{Growth rates in $\mathrm{NaCl}$ and glucose solutions}

For growth rate measurement, $6 \mathrm{ml}$ of freshly prepared Sabouraud dextrose (SD) broth was added in 3 sterile test tubes. Each test tube was made up to $10 \mathrm{ml}$ with final concentrations of $0.1 \mathrm{M}, 0.2 \mathrm{M}$ and $0.5 \mathrm{M} \mathrm{NaCl}$ respectively. Exactly $0.1 \mathrm{ml}$ of $10^{4} \mathrm{CFU}$ of the identified $C$. tropicalis isolate was inoculated into each test tube and incubated overnight $(\mathrm{O} / \mathrm{N})$ at $37^{\circ} \mathrm{C}$. A similar experimental set up containing $10 \mathrm{ml}$ of $\mathrm{SD}$ broth without $\mathrm{NaCl}$ solution was prepared as the control and inoculated with $10^{4} \mathrm{CFU}$ of cells, the culture was incubated $\mathrm{O} / \mathrm{N}$ at $37^{\circ} \mathrm{C}$. At time intervals of $0,3,6$ and $9 \mathrm{~h}, 2 \mathrm{ml}$ of the content of each test tube was placed into a cuvette and the quantity of $C$. tropicalis cells in the solution was determined using spectrophotometer (UNICO ${ }^{\circledR}$ series spectrophotometer) at $600 \mathrm{~nm}$. The growth rate assay was repeated at the same time intervals using $0.2,0.5$ and $1 \mathrm{~g} / \mathrm{ml}$ glucose concentrations with a control group containing SD broth without glucose. There were 3 biological replicates for each experimental set up.

\section{Preparation of plant extracts}

Leaves of $B$. coccineus Schum. and Thonn. was harvested from Orba in Udenu Local Government Area of Enugu state, Nigeria. The plant was identified by Mr. A.O. Ozioko, a plant taxonomist at the International Center for Ethno-medicine and Drug Development, Nsukka (ICEMDD). The leaves were rinsed with running tap water and air-dried on a clean surface at room temperature for 7 days. The dried leaves were then pulverized with an electric blender to obtain a fine powder. Exactly $500 \mathrm{~g}$ of the pulverized leaves were subjected to extraction using soxhlet extractor (Electrothermal heating mantle, model MS-9506). The samples were packed into the soxhlet column to $2 / 3$ of its volume and filled with $95 \%$ methanol, the extraction solvent. The soxhlet was then placed on the heating mantle adjusted to $40^{\circ} \mathrm{C}$. The methanol was allowed to reflux repeatedly, until it was clear and free from the extracts. The extracted content was then subjected to rotary evaporator (Bibby sterlin Ltd, England, RE.200) until the methanol was completely evaporated to get the solidified crude extracts. The crude extract obtained was stored in sterilized amber coloured bottles and maintained at $4^{\circ} \mathrm{C}$ in a refrigerator. The extraction process was repeated with ethyl acetate as the extraction solvent.

\section{Antifungal susceptibility test}

One isolate from each of the five Candida spp identified on the $\mathrm{CHROM}$ agar Candida, including the isolate identified by molecular sequencing, were selected for the susceptibility study. Exactly 0.5 $\mathrm{ml}$ of $\mathrm{O} / \mathrm{N}$ cultures of each Candida isolate containing $8.0 \times 10^{5}$ CFU was spread on freshly prepared SDA using a sterile spreader. In each petri plate containing the Candida culture, 4 holes (A, B, C and $D), 3 \mathrm{~mm}$ in size were made with sterile borer at each side of the plate, $4 \mathrm{~cm}$ from the center. The holes were inoculated with 0.1 $\mathrm{ml}$ each of $\mathrm{H}_{2} \mathrm{O}, 500 \mathrm{mg} / \mathrm{ml} \mathrm{B}$. coccineus extracts (methanol and ethyl acetate) and $500 \mathrm{mg} / \mathrm{ml}$ of fluconazole, respectively. The experiment was repeated with $300,200,100$ and $50 \mathrm{mg} / \mathrm{ml}$ each of $B$. coccineus extracts and fluconazole, respectively and the zones of inhibition were measured. There were 3 biological replications for each experimental set up.

\section{Statistical analysis}

Data was analyzed using descriptive statistics and represented as means and standard deviations. Statistical differences between means obtained from the zones of inhibition were determined using Mann-Whitney test.

\section{RESULTS}

\section{Fungal isolation and identification}

Based on differences in colour on CHROM agar Candida, 
Table 1. Candida species isolated from patients (child bearing mothers) with different clinical conditions.

\begin{tabular}{lcccccc}
\hline Condition & C. tropicalis & C. krusei & C. albican & C. parapsilosis & Variable $^{\text {a }}$ & Mixed $^{\text {b }}$ \\
\hline Malaria & 12 & 10 & - & 5 & - & 3 \\
Typhoid & - & 4 & 8 & - & 4 & 4 \\
Diabetes & 5 & 8 & - & 3 & - & 3 \\
Healthy & 4 & 3 & - & 2 & - & - \\
Total & 21 & 25 & 8 & 10 & 4 & 10 \\
\hline
\end{tabular}

Total number of Candida spp isolated from each treatment condition. The Candida spp were identified based on morphological appearance on CHROMagar Candida. $\mathrm{a}=$ observed colour on CHROMagar Candida representing more than one Candida species in the literature. $\mathrm{b}=$ mixed, Candida culture contaminated with more than one Candida species.

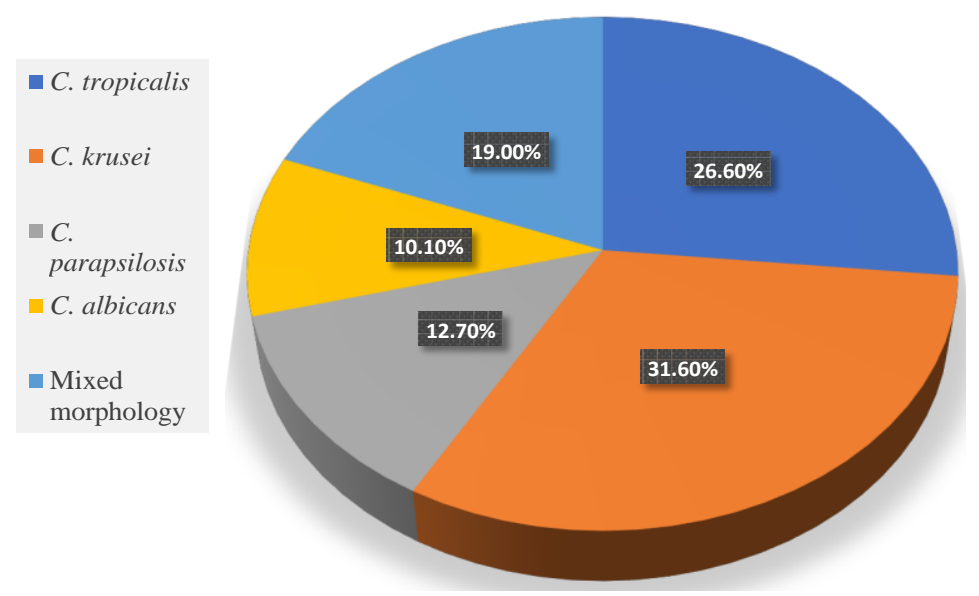

Figure 1. The percentage distribution of Candida spp isolated from this study. Percentages were calculated from the total number of each Candida species isolated from each of the treatment conditions.

the fungal isolates were separated into different Candida species. Out of the 100 patients sampled, 21 showed no growth of Candida and were dropped from further analysis. About 79 samples showed growth of Candida, $21(26.6 \%)$ were C. tropicalis, 25 (31.6\%) were C. krusei, $10(12.7 \%)$ were C. parapsilosis and $8(10.1 \%)$ were C. albicans (Table 1, Figure 1). Four samples appeared yellow on CHROM agar Candida and could not be classified because they exhibited variable status in the literature. Also, $15(19.0 \%)$ had mixed morphology on CHROM agar Candida and were not discernable. C. tropicalis and $C$. krusei were more prevalent in the malaria patients (Table 1), compared with the other categories. C. albicans was only found in the typhoid patients. About $8.9 \%$ of the women sampled in the study were in the control group.

\section{Molecular identification}

A BLASTN search of the NCBI database with the ITS sequence from the sequenced isolate revealed high sequence similarity $(100 \%$ identity, E-value $=0)$ to the sequence of $C$. tropicalis with accession number KF728820.1 (Figure 2). The phylogenetic analysis confirmed the identification of the isolate as $C$. tropicalis (Figure 2).

\section{Growth rates in glucose and $\mathrm{NaCl}$}

Including glucose in the growth medium reduced the growth of $C$. tropicalis (Figure 3 ). The biggest reduction in growth rate was found in the $1.0 \mathrm{~g} / \mathrm{ml}$ glucose solution (Figure 3), compared with the 0.2 and $0.5 \mathrm{~g} / \mathrm{ml}$ solutions. There were also observed differences in growth caused by addition of different concentrations of $\mathrm{NaCl}$ to the growth media. At 0 to $2 \mathrm{~h}$, the same growth pattern was observed in the 3 salt concentrations, but the fungus showed a slightly higher growth rate in the control media during this time points (Figure 4). At $3 \mathrm{~h}$, the fungus showed exponential growth in the medium with $0.2 \mathrm{M}$ and $0.3 \mathrm{M} \mathrm{NaCl}$ but the growth rate was drastically reduced by the $0.5 \mathrm{M}$ salt concentration. The highest growth rate 


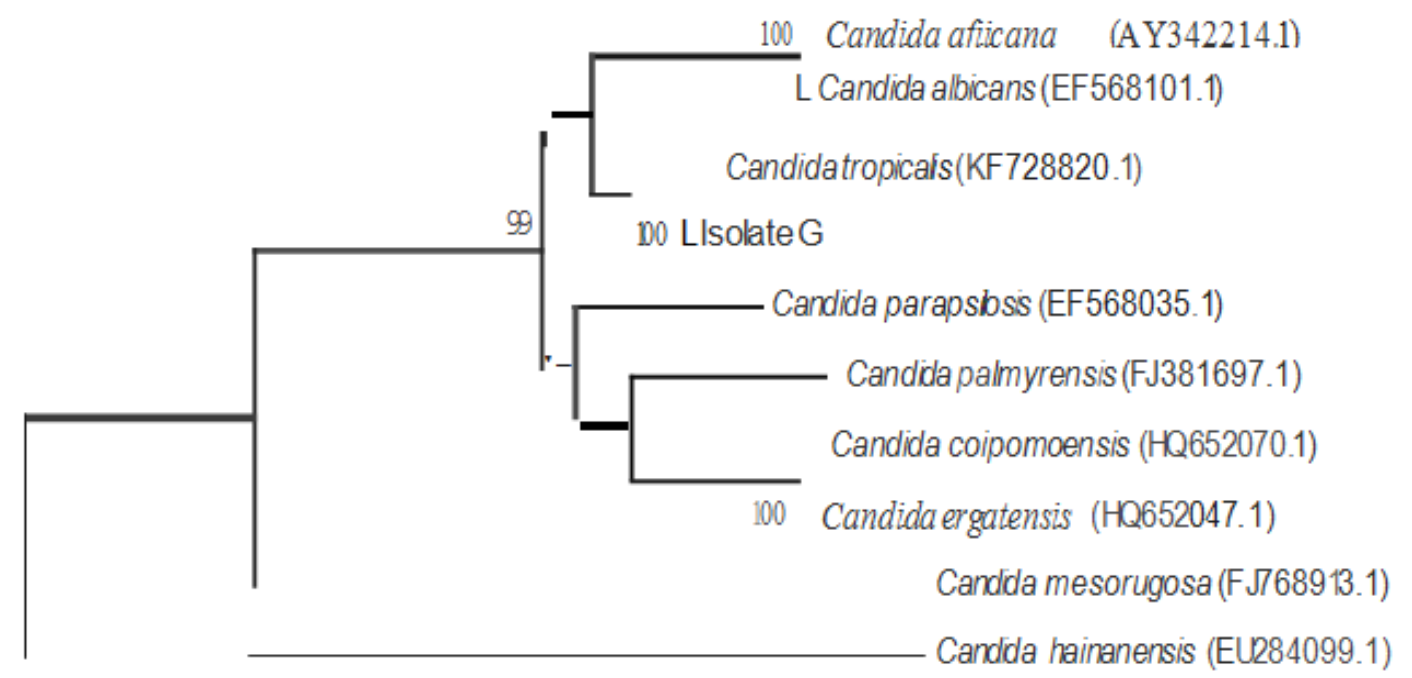

0.05

Figure 2. Evolutionary relatedness of the isolated Candida spp. Phylogenetic analysis was performed using neighbour-joining method implemented in MEGA5.0. Composite likelihood substitution model with uniform rates among sites and homogeneous patterns among lineages were employed in the analysis. Statistical support for the branching was determined by bootstrap analysis using 1000 replication. Branches without support are less than $70 \%$.

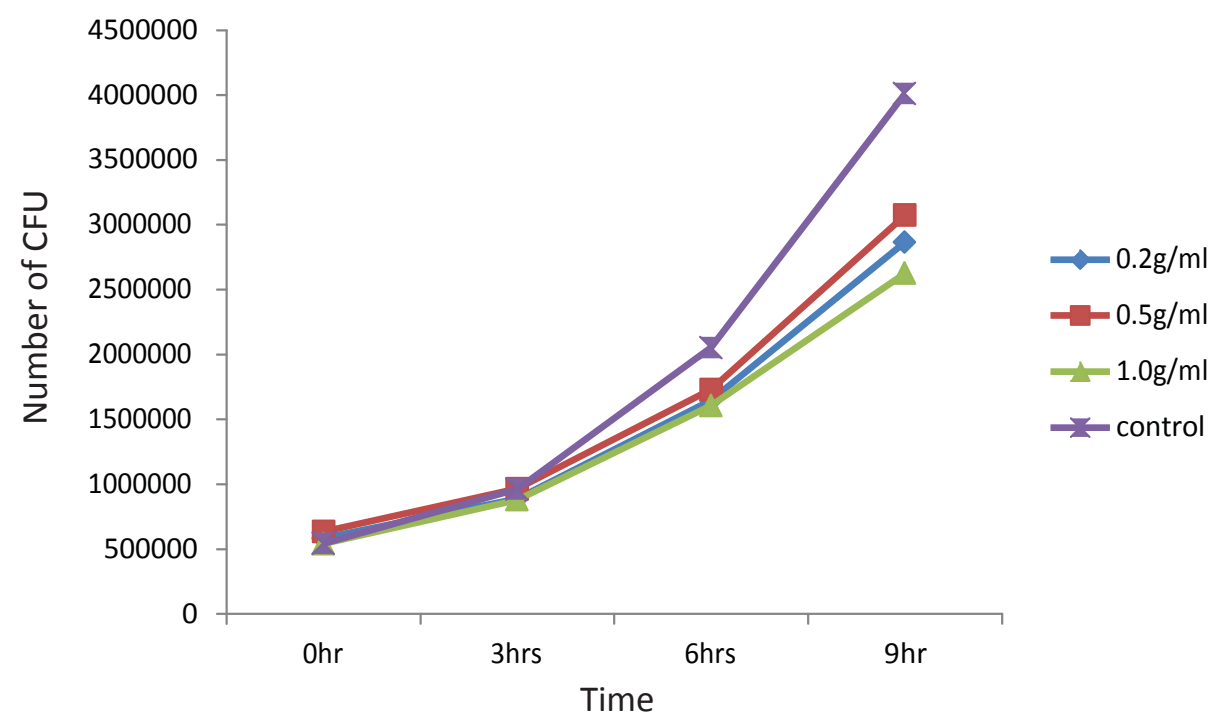

Figure 3. Growth curve of $C$. tropicalis in a glucose supplemented media. Growth rate was measured at time points $(0,3,6$ and $9 \mathrm{~h})$ in different glucose concentrations $(0.2,0.5$ and 1 $\mathrm{mg} / \mathrm{ml}$ ) by passing $2 \mathrm{ml}$ of the $\mathrm{O} / \mathrm{N}$ Candida culture through a spectrophotometer at $600 \mathrm{~nm}$.

was observed in the control medium (without $\mathrm{NaCl}$ ) at this time point. At $6 \mathrm{~h}$, a sharp decline was observed in the growth rate of the fungus in the control media while a plateau was evident in the 3 media with different salt concentrations.

\section{Susceptibility studies}

Fluconazole showed statistically significant higher activity than the two extracts from $B$. coccineus at any given concentration $(P=0.005)$. The zones of inhibition ranged 


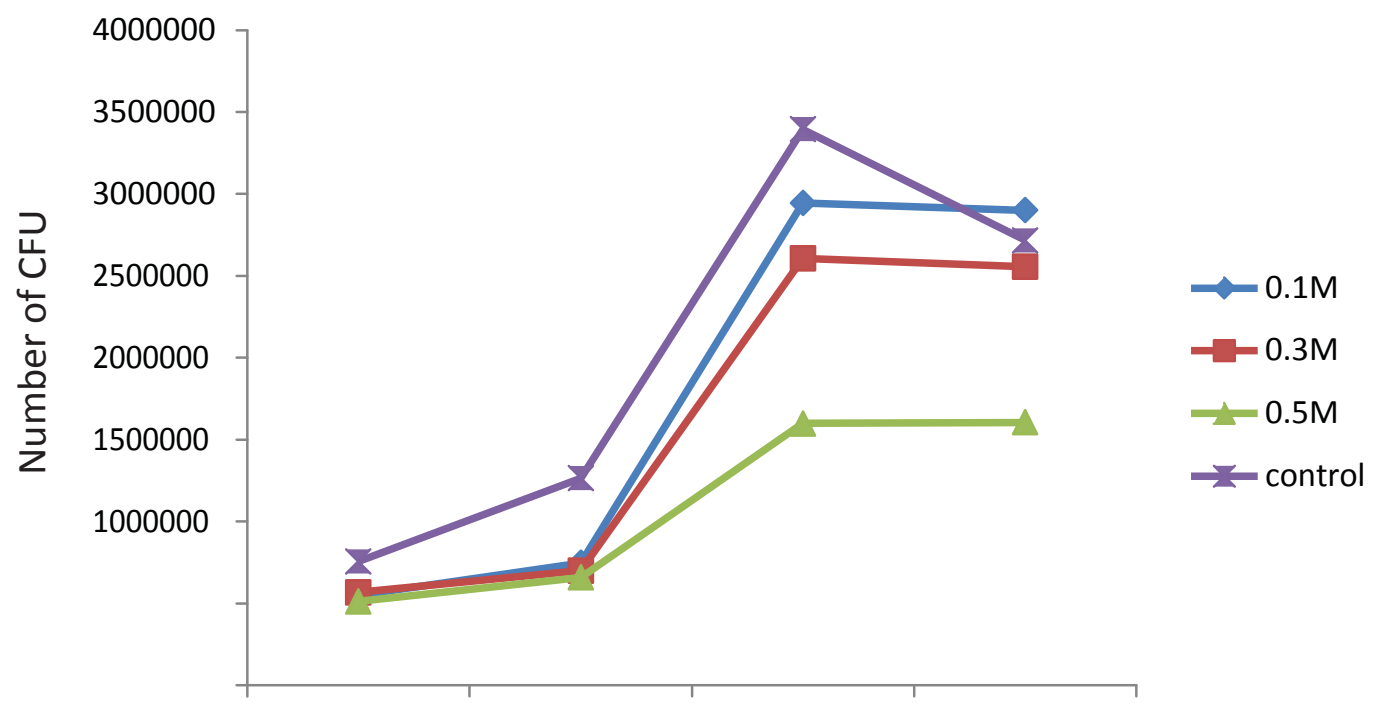

Figure 4. Growth curve of $C$. tropicalis in a salt supplemented media. Growth rate was measured at time points $(0,3,6$ and $9 \mathrm{~h})$ in different salt concentrations $(0.1,0.3$ and $0.5 \mathrm{M})$ by passing $2 \mathrm{ml}$ of the $\mathrm{O} / \mathrm{N}$ Candida culture through a spectrophotometer at $600 \mathrm{~nm}$.

Table 2. Susceptibility profile of the isolated Candida species to fluconazole, methanol and ethyl acetate extracts of $B$. coccineus.

\begin{tabular}{cccc}
\hline \multirow{2}{*}{ Concentrations (mg/ml) } & \multicolumn{3}{c}{ B. coccineus extracts } \\
\cline { 2 - 4 } & Fluconazole & Methanol extract & Ethyl extract \\
\hline 500 & $45.0 \pm 1.2^{\mathrm{a}}$ & $17.44 \pm 0.3^{\mathrm{b}}$ & $15.0 \pm 1.3^{\mathrm{b}}$ \\
200 & $40.44 \pm 3.4^{\mathrm{a}}$ & $14.22 \pm 1.1^{\mathrm{b}}$ & $14.78 \pm 0.6^{\mathrm{b}}$ \\
100 & $26.33 \pm 1.9^{\mathrm{a}}$ & $13.9 \pm 0.4^{\mathrm{b}}$ & $12.46 \pm 0.2^{\mathrm{b}}$ \\
50 & $31.83 \pm 2.1^{\mathrm{a}}$ & $14.22 \pm 0.7^{\mathrm{b}}$ & $12.33 \pm 1.7^{\mathrm{b}}$ \\
\hline
\end{tabular}

The susceptibility pattern was determined by measuring the zones of inhibition of fluconazole and the $B$. coccineus extracts against the growth of $8.0 \times 10^{5}$ inoculum of the Candida spp. Numbers with superscripts in each row indicate statistically significant difference at $0.05 \%$ level.

from 26.3 to $45.0 \mathrm{~mm}$ for fluconazole to 13.9 to $17.4 \mathrm{~mm}$ and 12.3 to $15.0 \mathrm{~mm}$ for the methanol and ethyl acetate extracts respectively (Table 2). Optimal activity of both fluconazole and the two extracts was observed at 500 $\mathrm{mg} / \mathrm{ml}$. At this concentration, the activity of the methanol extract was slightly higher than the activity of the ethyl acetate extract while the activity of the fluconazole control was almost twice the activities of the two extracts.

\section{DISCUSSION}

Candidiasis in women of child-bearing age is a major health problem in most hospitals especially among the immunocompromised patients (Lee et al., 2007). The distribution of Candida spp in vaginal candidiasis among women of child-bearing age under certain health conditions has not been fully investigated. In the present study, the prevalence of Candida in women 20 to 30 years of age who were suffering from malaria, diabetes and typhoid was compared. The antifungal susceptibility profile of the identified Candida species was also tested using both conventional azole and extracts from $B$. coccineus. Out of the 79 vaginal swabs (VGS) samples that showed presence of Candida, $C$. krusei had the highest occurrence $32.6 \%$, followed by $C$. tropicalis $26.6 \%$.

The occurrence of $C$. albicans and $C$. parapsilosis was relatively low 10.1 and $12.7 \%$, respectively. The normal/healthy patients presented low level of Candida colonization probably because of intact immune system. Most of the identified $C$. tropicalis were found in the malaria patients, but could not be statistically linked to malaria. Also, most of the $C$. albicans were isolated from the typhoid patients while $C$. krusei was the most abundant species in the patients with diabetes. However, 
C. albicans have been reported in other studies as the most abundant Candida species isolated from the vaginal swab of women with Type 1 diabetes, while C. glabrata was shown to be the most common isolate in Type 2 diabetic patients (Bohannon, 1998). Yeast infection in diabetic patients has been reported and Candida spp. described as the major infectious agent causing oral and esophageal candidiasis, pyelonephritis as well as cystitis (Casqueiro et al., 2012). In the present study, we did not classify the diabetic participants into Types 1 and 2 diabetics, it is possible that the variations in results could be as a result of differences in race or due to geographical variations. Studies have shown that the distribution of Candida species in women with vaginal candidiasis vary with geographic locations as well as the populations studied (Achkar and Fries, 2010). In the United States, Europe and Australia, C. albicans is the most abundant Candida species identified in 76 to $89 \%$ of studied women, followed by C. glabrata (7 to 16\%) (Corsello et al., 2003; Holland et al., 2003). Furthermore, in HIV infected patients, C. albicans and C. glabrata were found as the most prevalent Candida spp. colonizing the vaginal mucosa of infected patients (Merenstein et al., 2013). To further confirm the results from the morphologic appearance on CHROM agar Candida, we sequenced the ITS region of a randomly selected isolate that was presumptively identified as $C$. tropicalis based on its colour on CHROM agar Candida. BLASTN search of the sequenced isolate matched with the sequence of C. tropicalis in GenBank. CHROM agar Candida is a suitable media for the isolation and presumptive identification of Candida spp. based on strongly contrasted colony colours produced by reactions of species-specific enzymes with a chromogenic substrate (Khadka et al., 2016). CHROM agar Candida medium has been successfully used in the identification and differentiation of $C$. albicans, $C$. krusei, $C$. tropicalis, and Trichosporon spp. from other yeast species (Horvath et al., 2003).

Further analysis of the identified Candida spp. was done by tracing its evolutionary relatedness with other Candida spp. in the GenBank. Phylogenetic reconstruction showed one cluster separated into two small sub groups. The identified fungal isolate was more closely related to other pathogenic Candida spp. like $C$. tropicalis, C. albicans and $C$. africana in that order. The other small group comprised of mainly Candida spp. that have not been previously reported to be pathogenic in either humans or animals although $C$. parapsilosis which is a pathogenic species nested with this group. Other studies have demonstrated the close relationship between $C$. albicans and $C$. tropicalis using the internal transcribed spacer ITS1 gene (Butler et al., 2009). This intimate evolutionary relationship is also evident in phenotypic and biochemical characteristics of both species.

To determine how carbon and electrolyte sources affect the growth of the Candida spp, growth assays were carried out using graded levels of $\mathrm{NaCl}$ (table salt) and glucose. While lower concentrations of glucose in the medium $(0.2$ and $0.3 \mathrm{~g} / \mathrm{ml})$ increased the growth rate of the fungus, concentrations as high as $1 \mathrm{~g} / \mathrm{ml}$ adversely affected the growth rate. This result was not surprising as more energy and time may be required to metabolize the $1 \mathrm{~g} / \mathrm{ml}$ glucose concentration. Previous studies have shown that moderate carbon source modulates the physiological status of fungus and also plays a crucial role in fungus-host interaction (Ene et al., 2012a, b; Ueno et al., 2011; Vylkova et al., 2011). A similar trend was also observed in the behavior of the fungus in the presence of salt. While growth rate was improved in the presence of $0.2 \mathrm{M}$ and $0.3 \mathrm{M} \mathrm{NaCl}$ concentrations, the growth rate was reduced by $0.5 \mathrm{M} \mathrm{NaCl}$. Halotolerant yeasts have been shown to grow in the presence of salt concentrations as high as $4 \mathrm{M} \mathrm{NaCl}$ (Lages et al., 1999). In a survey performed on 42 different yeast species, Candida halophiles were the only yeasts capable of growing on glucose in the presence of $5 \mathrm{M} \mathrm{NaCl}$ (Lages et al., 1999). However, in our studies, growth rate was hampered by $0.5 \mathrm{M}$ concentration of the salt, an indication that the Candida spp. used in the growth assay is not a salt tolerant strain. We further analyzed the resistant pattern of the isolated Candida spp. using a conventional azole (fluconazole) and two extracts (methanolic and ethyl acetate) from B. coccineus. Fluconazole showed a higher activity than the two extracts with zone of inhibition as high as 26.3 to 45.0 $\mathrm{mm}$ compared to 13.9 to $17.4 \mathrm{~mm}$ and 12.3 to $15.0 \mathrm{~mm} \mathrm{ZI}$ for the methanol and ethyl acetate extracts respectively. Some Candida spp., such as C. glabrata and C. krusei have been shown to acquire decreased susceptibility to fluconazole (Cleveland et al., 2012; Pfaller et al., 2015). C. glabrata can survive in the hospital environment due to its high resistance to the commonly used azoles and echinocandins thereby increasing the chance of nosocomial transmission (Pfaller et al., 2011). For effective antifungal therapy therefore, the use of extracts from medicinal plants should be explored. However, our plant of choice $B$. coccineus showed decreased activity compared to the fluconazole. This could probably be as a result of the extraction methods or poor content of bioactive components in the leaves. The ethyl acetate extracts of $B$. coccinues has been found to have very high antimicrobial activity on C. albicans (Ahmadu et al., 2006). Also the butanolic extract of the plant has been shown to have high activity on $S$. aureus but the activity on C. albicans was quite low (Ahmadu et al., 2006). The antimicrobial activities of these extracts could be as a result of different bioactive components inherent in the leaves. Although we did not carry out phytochemical screening to investigate the bioactive components of the plant, other studies have reported the presence of tannins, saponins, sugars, coumeryl derivatives and flavonoid glycosides to be responsible for the antimicrobial 
activities (Ahmadu et al., 2006). In conclusion, although the extracts from $B$. coccineus showed a relatively lower activity compared to the fluconazole, it is a promising candidate for antifungal therapy. Additionally, C. tropicalis and C. krusei were the most common Candida spp. isolated from this study and they were more abundant in the malaria and diabetic patients respectively than in the other health conditions. Other Candida spp. like $C$. albicans and $C$. krusei were also isolated but at a relatively lower level.

\section{CONFLICT OF INTERESTS}

The authors have not declared any conflict of interests.

\section{ACKNOWLEDGEMENT}

We are sincerely grateful to Tertiary Education Trust Fund (TETFUND) for funding this project- Research project no. (TETFUND/DESS/UNI/NSUKKA/2017/RP/VOL.I). We also wish to thank the technical staff of the department of Microbiology, Faculty of Biological Sciences, University of Nigeria Nsukka for their assistance.

\section{REFERENCES}

Achkar JM, Fries BC (2010). Candida infections of the genitourinary tract. Clinical Microbiology Review 23:253-273.

Ahmadu AA, Akpulu NG, Hassan HS, Sule MI, Pate UU (2006). Preliminary phytochemical and antimicrobial screening of the leaves of Byrsocarpus coccineus Schum and Thonn (Connaraceae). Journal of Pharmacy and Bioresources 3:107-110.

Akindele AJ, Adeyemi OO (2006) Evaluation of Antidiarrheal activity of Brysocarpus coccineus. Journal of Ethnopharmacology 108(1):2025.

Alexander BD, Johnson MD, Pfeiffer CD, Jimenez-Ortigosa C, Catania J, Booker R, Castanheira M, Messer SA, Perlin DS, Pfaller MA (2013). Increasing echinocandin resistance in Candida glabrata: Clinical failure correlates with presence of FKS mutations and elevated minimum inhibitory concentrations. Clinical Infectious Disease 56(12):1724-1732.

Ben-Ami R (2018). Treatment of Invasive Candidiasis: A Narrative Review. Journal of Fungi (Basel) 4(3):97.

Bohannon NJ (1998). Treatment of vulvovaginal candidiasis in patients with diabetes. Diabetes Care 21:451-456.

Butler G, Rasmussen MD, Lin MF, Santos MA, Sakthikumar S, Munro CA, et al (2009). Evolution of pathogenicity and sexual reproduction in eight Candida genomes. Nature 459:657-662.

Canela HMS, Cardoso, Vitali LH, Coelho HC, Martinez R, Ferreira MEDS (2018). Prevalence, virulence factors and antifungal susceptibility of Candida spp. Isolated from bloodstream infections in a tertiary care hospital in Brazil. Mycoses 61:11-21.

Casqueiro J, Casqueiro J, Alves C (2012). Infections in patients with diabetes mellitus: a review of pathogenesis. Indian Journal of Endocrinology and Metabolism Supplement 1:S27-36.

Cleveland AA, Farley MM, Harrison LH, Stein B, Hollick R, Lockhart SR, Magill SS, Derado G, Park BJ, Chiller TM (2012). Changes in incidence and antifungal drug resistance in candidemia: Results from population-based laboratory surveillance in Atlanta and Baltimore, 2008-2011. Clinical Infectious Disease 55(10):1352-1361.

Cornely OA, Bassetti M, Calandra T, Garbino J, Kullberg BJ, Lortholary
O, Meersseman W, Akova M, Arendrup MC, Arikan-Akdagli S et al (2012) ESCMID guideline for the diagnosis and management of Candida diseases: Non-neutropenic adult patients. Clinical Microbiology and Infection 18:19-37.

Corsello S, Spinillo A, Osnengo G, Penna C, Guaschino S, Beltrame A Blasi N, Festa A (2003). An epidemiological survey of vulvovaginal candidiasis in Italy. European Journal of Biology 110:66-72.

Cruciani M, Serpelloni G (2008). Management of Candida infections in the adult intensive care unit. Expert Opinion in Pharmacotherapy 9:175-191.

Daef E, Moharram A, Seif Eldin S, Elsherbiny N, Mohammed M (2014). Evaluation of chromogenic media and semi nested PCR in the identification of Candida species. Brazilian Journal of Microbiology 45(1):255-262.

Denning DW, Kneale M, Sobel JD, Rautemaa-Richardson R (2018). Global burden of recurrent vulvovaginal candidiasis: a systematic review. Lancet Infectious Disease18:339-347.

Deorukhkar S, Saini S (2016). Echinocandin susceptibility profile of fluconazole resistant Candida species isolated from blood stream infections. Infectious Disorder and Drug Targets 16(1):63-68.

Deorukhkar S, Saini S, Mathew S (2014). Non-albicans Candida Infection: An emerging threat. Interdisciplinary Perspective on Infectious Diseases 2014:615958.

Deorukhkar SC, Saini S (2015). Candidiasis: Past, present and future. International Journal of Infection and Tropical Diseases 2:12-24.

Edgar RC (2004). MUSCLE: multiple sequence alignment with high accuracy and high throughput. Nucleic Acids Research 32:17921797.

Ene IV, Adya AK, Wehmeier S, Brand AC, Maccallum DM, Gow NA, Brown AJ (2012b). Host carbon sources modulate cell wall architecture, drug resistance and virulence in a fungal pathogen. Cell Microbiology 14:1319-1335.

Ene IV, Heilmann CJ, Sorgo AG, Walker LA, Koster CG, Munro CA, Klis FM, Brown AJ (2012a). Carbon source-induced reprogramming of the cell wall proteome and secretome modulates the adherence and drug resistance of the fungal pathogen Candida albicans. Proteomics 12:3164-3179

Fidel PL, Jr (2002). Immunity to Candida. Oral Diseases. 8: 69-75.

Gardes MI, Bruns TD (1993). ITS primers with enhanced specificity for basidiomycetes--application to the identification of mycorrhizae and rusts. Molecular Ecology 2:113-118.

Holland J, Young ML, Lee O, CA, Chen S (2003). Vulvovaginal carriage of yeasts other than Candida albicans. Sexually Transmitted Infection 79:249-250.

Horvath LL, Hospenthal DR, Murray CK, Dooley DP (2003). Direct Isolation of Candida spp. from blood cultures on the chromogenic medium CHROMagar Candida. Journal of Clinical Microbiology 41(6):2629-2632.

Kårén O, Högberg N, Dahlberg A, Jonsson L, Nylund JE (1997). Interand intraspecific variation in the ITS region of rDNA of ectomycorrhizal fungi in Fennoscandia as detected by endonuclease analysis. New Phytologist 136:313-325.

Khadka S, Regmi P, Giri S, Kumar Shah P, Kumar Mishra S (2016). Identification of Candida species using Chrom agar. International Journal of Medicine and Biomedical Sciences 1(3):10-13.

Kim J, Sudbery P (2011). Candida albicans, a major human fungal pathogen. Journal of Microbiology 49: 171.

Kossivi D, Tchazou K, Yao A, Kokou I, Amegnona A, Messanvi G (2014). Anti-inflammatory effect of Byrsocarpus coccineus Schum. and Thonn. (Connaraceae) root. World Journal of Pharmaceutical Research 3(3):3585-3598.

Lages F, Silva-Graca M, Lucas C (1999). Active glycerol up-take is a mechanism underlying halotolerance in yeasts: a study of 42 species. Microbiology 145:2577-2586.

Lee SA, Jones J, Khalique Z, Kot J, Alba M, Bernardo S, Seghal A, Wong B (2007). A functional analysis of the Candida albicans homolog of Saccharomyces cerevisiae VPS4. FEMS Yeast Research 7:973-985.

Merenstein D, Hu H, Wang C, Hamilton P, Blackmon M, Chen H, Calderone R, Li D (2013). Colonization by Candida Species of the oral and vaginal mucosa in HIV-infected and noninfected women. AIDS Research and Human Retroviruses 29:30-34. 
Murray PR, Baron EJ, Pfaller MA, Tenover FC, Yolken RH (2002). Manual of clinical microbiology ASM press. Washington D.C.

Muzny CA, Schwebke JR (2015). Biofilms: an underappreciated mechanism of treatment failure and recurrence in vaginal infections. Clinical Infectious Disease 61:601-602.

Nygren CMR, Eberhardt U, Karlsson M, Parrent JL, Lindahl BD, Taylor AFS (2008). Growth on nitrate and occurrence of nitrate reductaseencoding genes in a phylogenetically diverse range of ectomycorrhizal fungi. New Phytologist 180:875-889.

Pereira GH, Mu"ller PR, Szeszs MW, Levin AS, Melhem MS (2010). Five-year evaluation of bloodstream yeast infections in a tertiary hospital: the predominance of non- $C$. albicans Candida species. Medical Mycology 48: 839-842.

Pfaller MA, Moet GJ, Messer SA, Jones RN, Castanheira M (2011). Candida Bloodstream Infections: Comparison of Species Distributions and Antifungal Resistance Patterns in Community-Onset and Nosocomial Isolates in the SENTRY Antimicrobial Surveillance Program, 2008-2009. Antimicrobial agents and chemotherapy 55(2):561-566.

Pfaller MA, Rhomberg PR, Messer SA, Jones RN, Castanheira M (2015). Azaconazoles, micafungin, and 8 comparator antifungal agents' susceptibility profiles for common and uncommon opportunistic fungi collected in 2013: temporal analysis of antifungal drug resistance using CLSI species-specific clinical breakpoints and proposed epidemiological cutoff values. Diagnostic Microbiology and Infectious Disease 82(4):303-313.

Shao LC, Sheng CQ, Zhang WN (2007) Recent advances in the study of antifungal lead compounds with new chemical scaffolds. Yao Xue Xue Bao 42:1129-1136 (in Chinese).

Silva S, Negri M, Henriques M, Oliveria R, Williams DW, Azeredo J (2012). Candida glabrata, Candida parapsilosis and Candida tropicalis: biology, epidemiology, pathogenicity and antifungal resistance. FEMS Microbiology Review 36(2):288-305.
Tamura K, Peterson D, Peterson N, Stecher G, Nei M, Kumar S (2011) MEGA 5: molecular evolutionary genetics analysis using maximum likelihood, evolutionary distance and maximum parsimony methods. Molecular Biology and Evolution 28:2731-2739.

Ueno K, Matsumoto Y, Uno J, Sasamoto K, Sekimizu K, Kinjo Y, Chibana H (2011). Intestinal resident yeast Candida glabrata requires Cyb2p-mediated lactate assimilation to adapt in mouse intestine. PLoS One 6:e24759.

Vermitsky JP, Self MJ, Chadwick SG, Trama JP, Adelson ME, Mordechai E, Gygax SE (2008). Survey of vaginal-flora Candida species isolates from women of different age groups by use of species-specific PCR detection. Journal of Clinical Microbiology 46:1501-1503.

Vylkova S, Carman AJ, Danhof HA, Collette JR, Zhou H, Lorenz MC (2011). The fungal pathogen Candida albicans autoinduces hyphal morphogenesis by raising extracellular $\mathrm{pH}$. mBio 2(3):e00055-11.

White TJ, Bruns TD, Lee SB, Taylor JW (1990) Amplification and direct sequencing of fungal ribosomal RNA genes for phylogenetics. In: Innis MA, Gelfand DH, Sninsky JJ, White TJ, (editors). PCR protocols: a guide to methods and applications. Academic Press, San Diego, CA 18(1):315-322. 